\title{
When Will We Agree on the Origin and Meaning of 'Wellbeing'?
}

\author{
Lewis S Blevins, MD¹ and Robert Knutzen, $\mathrm{MBA}^{2}$
}

1. Clinical Professor of Neurological Surgery and Medicine and Medical Director, California Center for Pituitary Disorders, University of California San Francisco; 2. President and CEO, Pituitary Network Association, Thousand Oaks, California, US

\begin{abstract}
Quality of life is a nebulous term that is applied in medicine to assess the impact of disease states on the overall health of affected patients. It is difficult to measure, subject to interpretation based on the relative experiences of those assessing and reporting it, and there are no assessments or tools available to describe or determine the relative contributions of particular disease states and the affected person's constitution and pre-morbid state or personality to their quality of life.
\end{abstract}

\section{Keywords}

Quality of life, hormonal disorders, pituitary gland, acromegaly, Cushing's disease

Disclosure: The authors have no conflicts of interest to declare.

Received: December 2, 2011 Accepted: January 23, 2012 Citation: US Endocrinology, 2012;8(1):10-1 DOI: 10.17925/USE.2012.08.01.10

Correspondence: Lewis S Blevins, MD, California Center for Pituitary Disorders at UCSF, 400 Parnassus Avenue Room A-808, San Francisco, CA 94143-0350, US.

E: BlevinsL@neurosurg.ucsf.edu

'Quality of life', we believe, is becoming a more esoteric and often confusing term than first intended when it was originated. At one time just being alive constituted a primitive form of 'quality of life'.

As early as 1913, Dr Harvey Cushing, a premier neurosurgeon who was well ahead of his time, uttered the immortal words, "The psychopathology of everyday life most often hinges upon the discharge of the 'ductless' gland upon the nervous system." ${ }^{\text {1 }}$ His comments referred to substances, which we now recognise to be hormones that control other glands, produced by the anterior pituitary gland. More importantly, however, he recognised the association between disease states and what we now recognise to be a component of the gestalt of 'quality of life'. Advances in knowledge in the medical community since that time, and especially the development of 'instruments' to measure quality of life for various disease states, gave rise to both confusion and denial in regard to the relevance and importance of such information, most often adversely affecting the patient(s) in question.

In 1999, the National Institute of Mental Health, US Department of Health and Human Services, issued the report Mental Health: A Report of the Surgeon General. ${ }^{2}$ In the report, the then Secretary of Health and Human Services, Donna E Shalala, noted, "The United States leads the world in understanding the importance of overall health and wellbeing to the strength of a Nation and its people. What we are coming to realize is that mental health is absolutely essential to achieving prosperity." Subsequently, she related, "This Surgeon General's Report on Mental Health takes the next step in advancing the important notion that mental health is fundamental health." ${ }^{2}$ The report also points out the following: "Nearly two thirds of all people with diagnosable mental disorders do not seek treatment (Regier et al., 1993; Kessler et al., 1996). Stigma surrounding the receipt of mental health treatment is among the many barriers that discourage people from seeking treatment (Sussman et al., 1987; Cooper-Patrick et al., 1997)."3 In many ways, linking wellbeing to mental health was a setback, as the vast majority of patients who are ill, for one reason or another, do not have mental illness but rather psychological consequences of their underlying disorders. Further, the report explains that "the mental health field was adversely affected when underlying causes and treatments for particular disorders were identified. These advances resulted in patients with certain conditions, which adversely affect sense of wellbeing and mental health, being transferred for care from the mental health field to another medical specialty (Grob, 1991)."

For instance, dominion over syphilis was moved to dermatology, internal medicine and neurology upon advances in aetiology and treatment. Dominion over hormone-related disorders was moved into the realm of endocrinology. Was the field of endocrinology prepared and trained to deal with this new and largely unexplored and untested field of medicine? Was the field of endocrinology sufficiently aware of its responsibilities for the doubled task of assisting patients through both the physiological and emotional 'minefields' of what we now recognise as the total care of the patient? Some would argue that, regardless of specialty, too little attention is paid to the psychological consequences of diseases. A disease is nothing but a pathologic entity that affects an organ, or organ system, and thereby has consequences regarding the physiological health of the individual. We recognise illness as the complete manifestation of the disorder, encompassing physiological and psychological manifestations within the affected 
patient, and secondary psychological effects on family members, friends, co-workers, etc. Wellbeing, then, is more related to the illness than it is to the underlying disease process. Because of the complexities and the far-reaching effects of the illness, a sense of wellbeing is rather difficult to quantify, much less treat.

It is rapidly becoming an accepted fact that patients with pituitary and other hormonal disorders have long-lasting and difficult-to-control (or difficult-to-treat) psychological disturbances related to acute and chronic primary and secondary effects if there are hormonal perturbations. These too often bedevil their recovery even after treatment of the underlying disease process has been successful. Regrettably, many healthcare providers have deluded themselves into clinging to the belief that the patient's mental aberrations and 'misbehaviour' or 'reactive' depression originates after a diagnosis of a pituitary tumour or hormonal imbalance. It is often believed that psychological problems will resolve with treatment of the underlying disorder. Several studies have, however, illustrated that many of the problems, though they improve with therapy, do not fully resolve and patients are left with residual mood disorders, memory problems, sexual dysfunction, etc. Unfortunately, however, it is not often recognised that the affected patients may have displayed all the emotional/mental imbalances associated with emotional stress and disorder long, often years, before seeing an endocrinologist or neurosurgeon. Our point is that wellbeing can be affected by a disease process, an illness is modulated by treatment and recovery from such, and disturbances may persist after successful treatment.

How is 'wellbeing' to be defined? And once it is defined, how do we measure it and follow its course with treatment? How does one separate out pre-existing psychopathology from the psychological aberrations caused by an endocrine disorder? How do we remove confounding variables so that we can truly assess responses to treatment? We must strive to answer these questions to assist our patients and their families, who demand explanations, in understanding the rollercoaster effect of hormonal imbalances and how they cause aberrant behaviour or change the personality of affected people.

Even though they have a relatively long medical history, terms such as Cushing's disease, acromegaly, hyperprolactinemia, hyper- or hypopituitarism are not part of daily understanding or medical conversations as are cancer, glaucoma, cardiovascular disease, etc. Though most often neither thought of nor diagnosed, these conditions are very common and cause untold suffering, morbidity and, if untreated, mortality. In the plethora of clinical symptoms, the psychological state of the patients is too often overlooked or underexplored by a physician looking for an adenoma or even a hormonal imbalance. For instance, Cushing's disease is most often accompanied by depression, often for years and, as late as in the 1930s, suicide was a frequent cause of death in these patients. Acromegaly patients very often harbour anger, resentment, and/or aggressive behaviour, now often called 'acromegalic rage'. This is described by Paul Frederic Brain in a book chapter published in 1994 titled Hormonal aspects of aggression and violence. ${ }^{5}$ Hyperprolactinemic patients literally 'suffer' from severe mood swings (perhaps somewhat like those in premenstrual syndrome) and are simply unable to deliberately control their emotions, which can range from tearful 'blues' to short-tempered outbursts of anger. Hence, all three categories of diseases present the patients in an often antisocial light to the clinician as well as their immediate circle of friends and family. Clearly the patients suffer a severe decline in their 'psychological' quality of life, often over many years, and it adds insult to injury to not be recognised and validated by healthcare providers, who often associate this with a short-term 'upset' over a current or recent diagnosis of a hormonal disorder of some intensity.

Please recognise that each category of pituitary or other hormonal disorders may well present with a much larger range of emotional/psychological issues than described above and that the severity or intensity may vary from patient to patient and display/appear in no particular order. Children with growth hormone deficiency, evidenced by short stature and untreated, for instance, most often show clear and unmistakable signs and symptoms of the emotional and psychological effects of short stature, often exacerbated by teasing or even 'bullying' by their peers and classmates. Their psychological quality of life may, in many cases, suffer and linger for a lifetime, often requiring counselling and care to regain their emotional equilibrium.

In short, the psychology of quality of life is clear and unmistakable in most 'hormonally challenged' patients. However, the condition is rarely recognised for its medical origins but too often dismissed or ignored by the healthcare provider as a temporary aberration brought about by the shock of a tumour growth or a temporary but treatable hormonal balance disorder.

Further confusing the matter, quality of life is in the eye of the beholder. Many affected patients have no complaints whatsoever about their sense of wellbeing or quality of life, whereas other similarly affected patients might report of a horrible quality of life due to the same disease state. Quality of life, therefore, comes from within, is based upon a series of life experiences, expectations, relative comparisons, and attitudes towards health and wellbeing.

It may be well to heed the words of Plato, ${ }^{6}$ who said: "That as you ought not to attempt to cure the eyes without the head, or the head without the body, so neither ought you to cure the body without the soul", and this he said "is the reason why the cure of many diseases is unknown to the physicians of Hellas, because they are ignorant of the whole, which ought to be studied also; for the part can never be well unless the whole is well."
1. Falta W, The Ductless Glandular Diseases, Philadelphia: P. Blakiston's Son \& Co, 1915:51.

Shalala DE, A message from Donna Shalala. In: US Department of Health and Human Services, Mental Health: $A$ Report of the Surgeon General, Rockville, MD: US Department of Health and Human Services, Substance Abuse and Mental Health Services Administration, Center for Mental Health Services, National Institutes of Health, National Institute of Mental Health, 1999
3. US Department of Health and Human Services, Mental Health: A Report of the Surgeon General, Rockville, MD: US Department of Health and Human Services, Substance Abuse and Mental Health Services Administration, Center for Mental Health Services, National Institutes of Health, National Institute of Menta Health, 1999;8.

4. US Department of Health and Human Services, Mental Health: Report of the Surgeon General, Rockville, MD: US Department of
Health and Human Services, Substance Abuse and Mental Health Services Administration, Center for Mental Health Services, National Institutes of Health, National Institute of Mental Health, 1999:9.

5. Brain PF, Hormonal aspects of aggression and violence. In: Reiss AJ, Miczek KA, Roth JA (eds), Understanding and Preventing Violence - Vol 2: Biobehavioral Influences, Washington, DC: National Academy Press, 1994;173-244.

6. Plato, Charmides, or Temperance, 380 BCE;3. 